\title{
293.
}

\section{ON A CERTAIN SYSTEM OF FUNCTIONAL SYMBOLS.}

[From the Quarterly Journal of Pure and Applied Mathematics, vol. Iv. (1861), pp. $225 \div 230$.]

M. Hermite, in the Memoir "Sur la Résolution de l'équation du cinquième degré," Comptes Rendus, t. XLVI., (1858), p. 508, has given for equations of the fifth degree a solution by means of elliptic functions, analogous to the well-known trigonometrical solution of (the irreducible case of) the cubic equation. $\mathrm{He}$ has for this purpose to consider the effect of transforming certain functions $\phi \omega, \psi \omega$, connected with the elliptic functions, by replacing therein the argument $\omega$ by a linear fraction of the form $\frac{c+d \omega}{a+b \omega}$, where $a, b, c, d$ are integer numbers satisfying the condition

$$
a d-b c=1
$$

and he finds for $\phi\left(\frac{c+d \omega}{a+b \omega}\right)$ (and the like would apply to $\psi\left(\frac{c+d \omega}{a+b \omega}\right)$ ), six different expressions according to the evenness, or values in relation to the modulus 2 , of the coefficients $a, b, c, d$, viz., the congruence

$$
a d-b c \equiv 1(\bmod .2)
$$

admits of the six solutions shown by the table

\begin{tabular}{|c|c|c|c|c|}
\hline I. & 1 & 0 & 0 & 1 \\
\hline II. & 0 & 1 & 1 & 0 \\
\hline III. & 1 & 1 & 0 & 1 \\
\hline IV. & 1 & 1 & 1 & 0 \\
\hline V. & 1 & 0 & 1 & 1 \\
\hline VI. & 0 & 1 & 1 & 1 \\
\hline
\end{tabular}


(where 0 or 1 denotes that the coefficient to which it refers is even or odd), and to each solution there corresponds a distinct value of $\phi\left(\frac{c+d \omega}{a+b \omega}\right)$ or $\psi\left(\frac{c+d \omega}{a+b \omega}\right)$.

It appeared to me that the formulæ in question were not only of the highest importance in the theory of elliptic functions, but that they were very remarkable in and for themselves, and that the question might be looked at in the following manner.

Suppose that $A, B$ are functional symbols, or, so to term them, substitutions, the laws of operation being

$$
\begin{aligned}
& A(X, Y)=\left(\theta \frac{X}{Y}, \frac{1}{Y}\right), \\
& B(X, Y)=(Y, X),
\end{aligned}
$$

where $\theta$ is a constant. The equations mean that the effect of $A$ is to change $X$ into $\theta \frac{X}{Y}$, and $Y$ into $\frac{1}{Y}$, and the effect of $B$ to change $X$ into $Y$, and $Y$ into $X$. We have

$$
\begin{aligned}
& A^{2} X=A \cdot \theta \frac{X}{\bar{Y}}=\theta \frac{A X}{A Y}=\theta \cdot \theta \frac{X}{Y} \div \frac{1}{Y}=\theta^{2} X \\
& A^{2} Y=A \quad \frac{1}{Y}=\frac{1}{A Y}=\quad 1 \div \frac{1}{Y}=Y, \quad \text { or } A^{2}(X, Y)=\left(\theta^{2} X, Y\right),
\end{aligned}
$$

and so

$$
B^{2} X=X, \quad B^{2} Y=Y, \quad \text { or } B^{2}(X, Y)=(X, Y) ;
$$

thus $B$ is periodic of the second order; and in the particular case $\theta=1, A$ is also periodic of the second order.

This being so, let $K$ denote any one of the compound symbols $A^{p}, B A^{p}, A^{p} B$, $A^{q} B A^{p}$, \&c.; I say that the species of $K$ is determined by connecting with each of the symbols in question, a certain continued fraction as follows:

with

$$
\begin{array}{c|c}
A, p+\omega, & A^{p} B,-\frac{1}{p+\omega}, \\
B A^{p}, p-\frac{1}{\omega}, & B A^{p} B,-\frac{1}{p-\frac{1}{\omega}}, \\
A^{q} B A^{p}, p-\frac{1}{q+\omega}, & A^{q} B A^{p} B,-\frac{1}{p-\frac{1}{q+\omega}} \\
B A^{q} B A^{p}, p-\frac{1}{q-\frac{1}{\omega}}, & \& c ., \\
A^{r} B A^{q} B A^{p}, p-\frac{1}{q-\frac{1}{r+\omega}} & \\
\& c ., &
\end{array}
$$


and when the continued fraction is reduced to the form $\frac{c+d \omega}{a+b \omega}$, where, of course, $a d-b c=1$, then, that the corresponding symbol $K$ is of the species I. if $a, b, c, d \equiv 1,0,0,1$ (mod. 2), and in like manner, that the species is II., III., IV., V., or VI., according as $a, b, c, d$ are in regard to the modulus 2 of the form specified in the second, third, fourth, fifth, or sixth line of the foregoing table.

We have then a system of equations which I write in the form

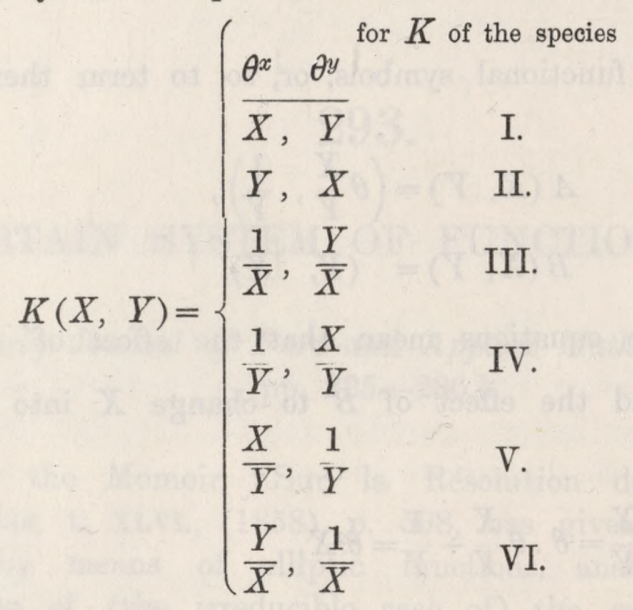

viz., the first line denotes that $K(X, Y)=\left(\theta^{x} X, \theta^{y} Y\right)$, and so for the other lines, the values of the indices $x, y$, being different in the several cases, but $\mathbf{I}$ bave written the system in the above form, to put in evidence more distinctly the theorem as applied to the most simple case, where $\theta=1$.

It will be sufficient to indicate how the theorem is proved in this particular case: I will suppose that for

we have

$$
K=B A^{q} B A^{p} \ldots,
$$

$$
K(X, Y)=\left\{\begin{array}{llc}
\multicolumn{3}{c}{\text { for } K \text { of the species }} \\
Y, & Y & \text { I. } \\
\frac{1}{X}, & \frac{Y}{X} & \text { II. } \\
\frac{1}{Y}, \frac{X}{Y} & \text { III. } \\
\frac{X}{Y}, \frac{1}{X} & \text { IV. } \\
\frac{Y}{X}, \frac{1}{X} & \text { VI. }
\end{array}\right.
$$

and let it be inquired what is the effect of the symbol $K^{\prime}=A^{r} B A^{q} B A^{p} \ldots$, which, since $A^{2}(X, Y)=(X, Y)$, is equivalent to $A K$ or $K$, according as $r$ is odd or even. 
Let $\Theta=\frac{c+d \omega}{a+b \omega}$ be the continued fraction which corresponds to $K$, and $\Theta^{\prime}=\frac{c^{\prime}+d^{\prime} \omega}{a^{\prime}+b^{\prime} \omega}$ the continued fraction which corresponds to $K^{\prime}$, then $\Theta^{\prime}$ is deduced from $\Theta$ by putting therein $r+\omega$ for $\omega$, or we have

$$
\frac{c+d(\omega+r)}{a+b(\omega+r)}=\frac{c^{\prime}+d^{\prime} \omega}{a^{\prime}+b^{\prime} \omega},
$$

or

$$
\begin{aligned}
& c^{\prime}=c+d r, d^{\prime}=d, \\
& a^{\prime}=a+b r, b^{\prime}=b,
\end{aligned}
$$

and therefore when $r$ is even, or $\equiv 0$ (mod. 2), then

$$
\begin{aligned}
& c^{\prime} \equiv c, d^{\prime} \equiv d, \quad(\bmod .2), \\
& a^{\prime} \equiv a, b^{\prime} \equiv b,
\end{aligned}
$$

or $K^{\prime}$ is of the same species as $K$. But in this case since $A^{2}(X, Y)=(X, Y)$ and $r$ is even, we ought to have $K^{\prime}$ equivalent to $K$; and hence $r$ being even, the theorem, assumed to be true for $K$, is also true for $K^{\prime}$.

But if $r$ be odd, or $\equiv 1$ (mod. 2$)$, then

$$
\begin{aligned}
& c^{\prime} \equiv c+1, d^{\prime} \equiv d, \quad(\bmod .2), \\
& a^{\prime} \equiv a+1, b^{\prime} \equiv b,
\end{aligned}
$$

whence, according as

$$
\begin{aligned}
& K \text { is of species I., II., III., IV., V., VI., so } \\
& K^{\prime} \ldots \ldots \ldots \text { V., IV., VI., II., I., III. }
\end{aligned}
$$

But if from $K(X, Y)$ we derive $A K(X, Y)$, we find that of whatever species $K$ may be, the species of $A K$ is identical with the corresponding species of $K^{\prime}$, and in the case in question, where $r$ is odd, it is clear that we ought to have $K^{\prime}$ equivalent to $A K$. Hence in this case also, and therefore generally, if the theorem be true for $K$ it will be also true for $K^{\prime}$.

In a very similar manner it may be shown that if the theorem is true for $K^{\prime}=A^{r} B A^{q} B A^{p} \ldots$, it will be true for $B A^{r} B A^{q} B A^{p} \ldots$, and the foregoing cases include the case where the symbol is of the form $B A^{r} B A^{q} B$ or $A^{r} B A^{q} B$ (terminating in a $B$ ), for it is only necessary to assume that the last index of $A$ is zero; hence the theorem, if true in any case, as it obviously is for $A^{0}(X, Y)=(X, Y)$, is true universally.

When $\theta$ is not equal 1 , the only difference is that the terms of $K(X, Y)$ are respectively multiplied by certain powers $\theta^{x}, \theta^{y}$ of $\theta$, and it is not difficult to see that for $K=A^{r} B A^{q} B A^{p} \ldots$ the indices $x, y$ are of the forms

$$
\begin{aligned}
& x=\mathrm{c} r+\mathrm{b} q+\mathrm{a} p+\ldots, \\
& y=\mathrm{c}^{\prime} r+\mathrm{b}^{\prime} q+\mathrm{a}^{\prime} p+\ldots,
\end{aligned}
$$


where $c, b, a, \ldots, c^{\prime}, b^{\prime}, a^{\prime}, \ldots$ have only the values $0,+1,-1$, the values of

$$
\begin{aligned}
& \mathrm{c}, \mathrm{c}^{\prime} \text { depending on the species of } B A^{q} B A^{p} \ldots \\
& \mathrm{b}, \mathrm{b}^{\prime} \\
& \mathrm{a}, \mathrm{a}
\end{aligned}
$$

in a manner which can easily be determined. In the particular case, where $\theta^{16}=1$ (as it is in M. Hermite's problem), it is only necessary to know the values of $x, y$ in regard to the modulus 16, and these values can be expressed in terms of the numbers $(a, b, c, d)$ which correspond to the symbol $K$ (this is in itself a remarkable theorem), and I find that (to modulus 16)

$$
x \equiv\left\{\begin{array}{l}
d(c+d)-1 \\
c(c-d)-1 \\
d(d-c)-1 \\
c(d-c)+1 \\
c d \\
-c d
\end{array} \quad y=\left\{\begin{array}{cc}
a(a-b)-1 & \text { for } K \text { of the species } \\
b(a+b)-1 & \text { II. } \\
a b & \text { III. } \\
-a b & \text { IV. } \\
a(b-a)+1 & \text { V. } \\
b(b-a)-1 & \text { VI. }
\end{array}\right.\right.
$$

This may be shown by induction in like manner as the theorem in regard to the parts independent of $\theta$. Thus $K, K^{\prime}$ as before, let $K$ be of the species I., and assume that $K(X, Y)=\left(\theta^{x} X, \theta^{y} Y\right)$ where

$$
x \equiv d(c+d)-1, y \equiv a(a-b)-1(\bmod .16),
$$

then if $r$ be even, since $A^{2}(X, Y)=\left(\theta^{2} X, Y\right)$, we ought to have $K^{\prime}(X, Y)=\left(\theta^{x+2 r} X, \theta^{y} Y\right)$. But in this case $K^{\prime}$ is of the same species with $K$, viz., of the species I., and by the theorem, $K^{\prime}(X, Y)=\left(\theta^{x^{\prime}} X, \theta^{y^{\prime}} Y\right)$ where

$$
x^{\prime} \equiv d^{\prime}\left(c^{\prime}+d^{\prime}\right)-1, y^{\prime} \equiv a^{\prime}\left(a^{\prime}-b^{\prime}\right)-1(\bmod .16) ;
$$

and we ought to have

that is

$$
\begin{aligned}
& x^{\prime} \equiv x+r, y^{\prime} \equiv y(\bmod .16) \\
& d^{\prime}\left(c^{\prime}+d^{\prime}\right)-1 \equiv d(c+d)-1+r,(\bmod .16), \\
& a^{\prime}\left(a^{\prime}-b^{\prime}\right)-1 \equiv a(a-b)-1
\end{aligned}
$$

or substituting for $a^{\prime}, b^{\prime}, c^{\prime}, d^{\prime}$ their values $a+b r, b, c+d r, d$, these become

$$
\begin{aligned}
d(c+d+d r) & \equiv d(c+d)+r,(\text { mod. } 16), \\
(a+b r)(a-b+b r) & \equiv a(a-b)
\end{aligned}
$$

or

$$
\begin{aligned}
\left(d^{2}-1\right) r & \equiv 0, \quad(\bmod .16) . \\
\left(2 a b-b^{2}+b^{2} r\right) r & \equiv 0
\end{aligned}
$$


But $r$ being even, these become

$$
d^{2}-1 \equiv 0,2 a b-b^{2}+b^{2} r \equiv 0(\bmod .8) ;
$$

or, since in the case under consideration, $a, b, c, d \equiv 1,0,0,1$ (mod. 2), and therefore $b^{2} r \equiv 0$ (mod. 8), the two congruences become

$$
d^{2}-1 \equiv 0, a^{2}-(a-b)^{2} \equiv 0(\bmod .8),
$$

which are satisfied since $d, a$, and $a-b$ are all odd. And in a similar way the theorem is proved for all the other cases.

In M. Hermite's problem, $X, Y$ are replaced by $\phi \omega, \psi \omega, A X, A Y$ are $\phi(\omega+1)$, $\psi(\omega+1)$, and $B X, B Y$ are $\phi\left(-\frac{1}{\omega}\right), \psi\left(-\frac{1}{\omega}\right)$, the properties of the functions $\phi, \psi$ being such that, as in the preceding investigation,

$$
A(X, Y)=\left(\theta \frac{X}{Y}, \frac{1}{Y}\right), \quad B(X, Y)=(Y, X)
$$

where $\theta\left(=e^{\frac{1}{2} i \pi}\right)$ is a sixteenth root of unity, and $K X, K Y$ are respectively equal to $\phi\left(\frac{c+d \omega}{a+b \omega}\right), \psi\left(\frac{c+d \omega}{a+b \omega}\right)$, and the formulæ are precisely similar to those of the present paper.

2, Stone Buildings, W.C., 5 May, 1860.

C. IV. 